\title{
HEATH $\boldsymbol{g}$-FUNCTIONS AND METRIZATION
}

\author{
ABDUL M. MOHAMAD \\ (Received 13 November 2002; revised 10 September 2003)
}

Communicated by S. Gadde

\begin{abstract}
In this paper, we present some new metrization theorems in terms of Heath $g$-functions.

2000 Mathematics subject classification: primary 54E30, 54E35.

Keywords and phrases: stratifiability, $w M$-space, Heath $g$-function, metrizability.
\end{abstract}

\section{Introduction}

In this short note we characterize metrizability in terms of Heath $g$-functions.

Heath in [3] introduced a method of describing a generalized metric property of a topological space $(X, \tau)$ by means of a function $g: \mathbb{N} \times X \rightarrow \tau$. Hodel, Fletcher, Lindgren and Nagata have modified this method to obtain important new classes of spaces.

A Heath $g$-function [COC-map (= countable open covering map)] for a topological space $X$ is a function $g$ from $\mathbb{N} \times X$ into the topology of $X$ such that for every $x \in X$ and $n \in \mathbb{N}, x \in g(n, x)$ and $g(n+1, x) \subseteq g(n, x)$.

It is well known that many important classes of generalized metrizable spaces can be characterized in terms of a Heath $g$-function. In particular, $X$ is developable [3] ( $w \Delta$-space) if and only if $X$ has a Heath $g$-function $g$ such that if $\left\{p, x_{n}\right\} \subseteq g\left(n, y_{n}\right)$ for all $n$, then $p$ is a cluster point of the sequence $\left\langle x_{n}\right\rangle$ (then $\left\langle x_{n}\right\rangle$ has a cluster point).

A space $X$ is called a $w \dot{M}$-space [4] if and only if $X$ has a Heath $g$-function $g$ such that if $x \in g\left(n, z_{n}\right), g\left(n, z_{n}\right) \cap g\left(n, y_{n}\right) \neq \emptyset$ and $x_{n} \in g\left(n, y_{n}\right)$ for all $n$ then $\left\langle x_{n}\right)$ has a cluster point. Let $\mathscr{G}$ be a collection of sets. We define $s t(x, \mathscr{G})=\bigcup\{G \in \mathscr{G}: x \in G\}$ and $s t^{2}(x, \mathscr{G})=\bigcup_{y \in s i(x, \mathscr{G})} s t(y, \mathscr{G})$.

In this paper all spaces will be $T_{0}$, unless we state otherwise.

(C) 2005 Australian Mathematical Society $1446-7887 / 05 \$ A 2.00+0.00$ 


\section{Main results}

First we consider what additional conditions need to be attached to a space already known to be stratifiable (conditions (1)-(3) in the next theorem are the axioms of stratifiability [2, Theorem 5.8]) to make it metrizable. To do this, the function $g: \omega \times X \rightarrow \mathscr{T}$ can be strengthened to give it some sort of symmetry as shown by the next theorem.

THEOREM 2.1. A space $X$ is metrizable if and only if there exists a function $g: \omega \times X \rightarrow \mathscr{T}$ such that

(1) $\{x\}=\bigcap_{n \in \omega} g(n, x)$;

(2) if $y \in g\left(n, x_{n}\right)$ for all $n$ then $x_{n} \rightarrow y$;

(3) for any $y \notin H$ closed, $y \notin \overline{\bigcup\{g(x, n): x \in H\}}$ for some $n \in \omega$;

(4) if $y \in g(n, x)$ then $x \in g(n, y)$.

PROOF. For any metric space we can define $g$ to satisfy the axioms of stratifiability as given in [2, Theorem 5.8]. The fourth condition of the theorem holds because of the symmetry of a metric.

To prove the converse, we assume that without loss of generality,

$$
g(n, x) \subseteq g(n+1, x) \text { for any } x \in X .
$$

If this was not the case, we define the function $g^{\prime}(n, x)=\bigcap_{k \leqslant n} g(k, x)$. Certainly each $g^{\prime}(n, x)$ is open as the finite intersection of open sets and the axioms for stratifiability still hold since $g^{\prime}(n, x) \subseteq g(n, x)$ for each $n \in \omega$ and $x \in X$. Notice also that condition (4) remains true when considering these new open sets.

$X$ can be shown to be a $T_{1}$ space by showing $\{x\}$ is closed for each $x \in X$. Suppose $y \notin\{x\}$; that is, $y \neq x$. Then we must have some $n \in \omega$ such that $x \notin g(n, y)$, otherwise $x \in \bigcap_{n \in \omega} g(n, y)=\{y\}$ and so the points are not distinct. Hence there is an open neighbourhood of $y$ which does not meet $\{x\}$ and so $\{x\}$ is closed.

For each $n \in \omega$, we define an open cover $\mathscr{G}_{n}=\{g(n, x): x \in X\}$. Suppose that $x$ is in some open set $U$. If we can show that there exists some $n \in \omega$ such that $s t^{2}\left(x, \mathscr{G}_{n}\right) \subseteq U$ then since $X$ is $T_{0}$, the space will be metrizable by the Moore Metrization theorem [1].

Firstly we notice that there must exist some $n_{0} \in \omega$ such that $g\left(n_{0}, x\right) \subseteq U$, otherwise we can define a sequence of points $x_{n}$ such that $x_{n} \in g(n, x) \backslash U$ for each $n \in \omega$. Then by our new symmetry condition, $x \in g\left(n, x_{n}\right)$ for each $n \in \omega$, hence $x_{n} \rightarrow x$, contradicting the fact that $x \in U$ since the points $x_{n}$ all lie in the closed set $X \backslash U$ and so their limit must also lie in $X \backslash U$. Define $U_{1}=g\left(n_{0}, x\right)$ and notice that

$$
x \notin X \backslash U_{1} \Rightarrow x \notin \overline{\bigcup\left\{g\left(n_{1}, y\right): y \in X \backslash U_{1}\right\}}=X \backslash U_{2}, \quad \text { some } n_{1} \in \omega,
$$




$$
\begin{aligned}
& x \notin X \backslash U_{2} \Rightarrow x \notin \overline{\bigcup\left\{g\left(n_{2}, y\right): y \in X \backslash U_{2}\right\}}=X \backslash U_{3}, \quad \text { some } n_{2} \in \omega, \\
& x \notin X \backslash U_{3} \Rightarrow x \notin \overline{\bigcup\left\{g\left(n_{3}, y\right): y \in X \backslash U_{3}\right\}}=X \backslash U_{4}, \quad \text { some } n_{3} \in \omega, \\
& x \notin X \backslash U _ { 4 } \Rightarrow x \notin \longdiv { \bigcup \{ g ( n _ { 4 } , y ) : y \in X \backslash U _ { 4 } \} } = X \backslash U _ { 5 }, \quad \text { some } n_{4} \in \omega .
\end{aligned}
$$

Let $n=\max \left\{n_{0}, n_{1}, n_{2}, n_{3}, n_{4}\right\}$. We now show that $s t\left(x, \mathscr{G}_{n}\right) \subseteq U_{3}$. If $x_{2}$ is any point in $\operatorname{st}\left(x, \mathscr{G}_{n}\right)$ then there is some $x_{1}$ such that $x \in g\left(n, x_{1}\right)$ and $x_{2} \in g\left(n, x_{1}\right)$, hence $x_{1} \in g\left(n, x_{2}\right)$ and $x_{1} \in g\left(n_{3}, x_{2}\right)$. If we assume that $x_{2} \notin U_{3}$, then $x_{2} \in X \backslash U_{3}$, so $x_{1} \in \overline{\bigcup\left\{g\left(n_{3}, y\right): y \in X \backslash U_{3}\right\}}=X \backslash U_{4}$. Similarly, since we have $x_{1} \notin U_{4}$ and $x \in g\left(n, x_{1}\right)$ (hence $\left.x \in g\left(n_{4}, x_{1}\right)\right)$, then $x \in \overline{U\left\{g\left(n_{4}, y\right): y \in X \backslash U_{4}\right\}}=X \backslash U_{5}$ which contradicts the fact that $x \in U_{5}$. This means that $x_{2} \in U_{3}$ and so st $\left(x, \mathscr{G}_{n}\right) \subseteq U_{3}$.

The final stage of the proof is to show that $s t^{2}\left(x, \mathscr{G}_{n}\right) \subseteq U$ by showing that $s t^{2}\left(x, \mathscr{G}_{n}\right) \subseteq U_{1}$. Consider $x_{4} \in s t^{2}\left(x, \mathscr{G}_{n}\right)$. This means we have some point $x_{3}$ such that $x_{2} \in g\left(n, x_{3}\right)$ and $x_{4} \in g\left(n, x_{3}\right)$ (for some $x_{2} \in$ st $\left(x, \mathscr{G}_{n}\right)$ ), hence $x_{3} \in g\left(n, x_{4}\right)$ and $x_{3} \in g\left(n_{1}, x_{4}\right)$. If we assume that $x_{4} \notin U_{1}$, then $x_{3} \in \overline{\bigcup\left\{g\left(n_{1}, y\right): y \in X \backslash U_{1}\right\}}=$ $\dot{X} \backslash U_{2}$. Similarly, since we have $x_{3} \notin U_{2}$ and $x_{2} \in g\left(n, x_{3}\right)$ (hence $x_{2} \in g\left(n_{2}, x_{3}\right)$ ), then $x_{2} \in \overline{\bigcup\left\{g\left(n_{2}, y\right): y \in X \backslash U_{2}\right\}}=X \backslash U_{3}$ which contradicts the fact that $x_{2} \in U_{3}$. This means that $x_{4} \in U_{1}$ and so $s t^{2}\left(x, \mathscr{G}_{n}\right) \subseteq U_{1} \subseteq U$.

We now consider some similar results where, instead of requiring convergence of sequences, we only require clustering.

THEOREM 2.2. A space $X$ is metrizable if and only if there is a Heath $g$-function $g$ such that

(1) if $x \in g(n, y)$ then $y \in g(n, x)$;

(2) if $\left\{x, x_{n}\right\} \subset g\left(n, y_{n}\right)$ for all $n$ then $x$ is a cluster point of the sequence $\left\langle x_{n}\right\rangle$.

Proof. Necessity is clear. For sufficiency: since the condition (2) gives developability to the space $X$, we need only to prove that $X$ is a regular and $w M$-space (every regular, developable, $w M$-space is metrizable [5]). We first prove $X$ is regular. Let $x \in U$ be open in $X$. Suppose $x_{n} \in \overline{g(n, x)}-U$ for all $n \in \mathbb{N}$. Then $y_{n} \in g(n, x) \cap g\left(n, x_{n}\right)$ for each $n$. So $x \in g\left(n, y_{n}\right)$ and $x_{n} \in g\left(n, y_{n}\right)$. Therefore, we have $\left\{x, x_{n}\right\} \subset g\left(n, y_{n}\right)$, so $x$ is a cluster point of the sequence $\left\langle x_{n}\right\rangle$. But $x \in U$ is open and $x_{n} \notin U$ for each $n$, which contradicts that $x$ is a cluster point of the sequence $\left\langle x_{n}\right\rangle$. Therefore, $\overline{g(n, x)} \subset U$ for some $n$ and $X$ is regular.

Finally, we prove $X$ is a $w M$-space. Let $x \in g\left(n, z_{n}\right), g\left(n, z_{n}\right) \cap g\left(n, y_{n}\right) \neq$ $\emptyset$ and $x_{n} \in g\left(n, y_{n}\right)$. Now we want to show that $\left\langle x_{n}\right\rangle$ has a cluster point. Let $p_{n} \in g\left(n, z_{n}\right) \cap g\left(n, y_{n}\right)$. Since $p_{n} \in g\left(n, z_{n}\right)$ and $x \in g\left(n, z_{n}\right),\left\{x, p_{n}\right\} \subset g\left(n, z_{n}\right)$. Therefore, $x$ is a cluster point of the sequence $\left\langle p_{n}\right\rangle$. There is a subsequence $\langle m(n)\rangle$ of the sequence $\langle n\rangle$ such that $p_{m(n)} \in g(n, x)$, which implies that $x \in g\left(n, p_{m(n)}\right)$. We 
have $p_{m(n)} \in g\left(n, y_{m(n)}\right)$, so $y_{m(n)} \in g\left(m(n), p_{m(n)}\right) \subset g\left(n, p_{m(n)}\right)$. Now $\left\{x, y_{m(n)}\right\} \subset$ $g\left(n, p_{m(n)}\right)$, so $x$ is a cluster point of the sequence $\left\langle y_{m(n)}\right\rangle$. Therefore, there is a subsequence $\langle m(n)(k)\rangle$ of the sequence $\langle m(n)\rangle$ such that $y_{m(n)(k)} \in g(k, x)$ for all $k$ and hence $x \in g\left(k, y_{m(n)(k)}\right)$ for all $k$. Since

$$
x_{m(n)(k)} \in g\left(m(n)(k), y_{m(n)(k)}\right) \subset g\left(k, y_{m(n)(k)}\right)
$$

$\left\{x, x_{m(n)(k)}\right\} \subset g\left(n, y_{m(n)(k)}\right)$ for all $k$ and hence $x$ is the cluster point of the sequence $\left\langle x_{m(n)(k)}\right\rangle$. Therefore, $x$ is the cluster point of the sequence $\left\langle x_{n}\right\rangle$.

We define $g^{1}(n, x)=g(n, x)$, and $g^{k+1}(n, x)=\bigcup\left\{g(n, y): y \in g^{k}(n, x)\right\}$ for $k \geq 1$.

THEOREM 2.3. A space $X$ is metrizable if and only if there is a Heath $g$-function $g$ such that

(1) if $x \in g(n, y)$ then $y \in g(n, x)$;

(2) if $x \in g^{2}\left(n, x_{n}\right)$ for all $n$ then $x$ is a cluster point of the sequence $\left\langle x_{n}\right\rangle$.

Proof. Let $X$ be metrizable space with a sequence $\left\{\mathscr{G}_{n}\right\}_{n \in \mathbb{N}}$ of open covers of $X$ satisfying that $\left\{s t^{2}\left(x, \mathscr{G}_{n}\right)\right\}$ is a local base at $x$ for all $x \in X$. Put $g(n, x)=s t\left(x, \mathscr{G}_{n}\right)$ for each $x \in X$ and for each $n$. Then $g$ is a COC-map which satisfies (1) and (2), because $g^{2}\left(n, x_{n}\right)=s t^{2}\left(x, \mathscr{G}_{n}\right)$.

For the converse, we can prove by induction on $k$ that if $\left\langle x_{n}\right\rangle$ is a sequence in $X$ and $x \in X$ with $x_{n} \in g^{k}(n, x)$ for all $n$ then $x$ is a cluster point of $\left\langle x_{n}\right\rangle$. From this it follows that if $U$ open with $x \in U$ then there is some $n$ with $g^{4}(n, x) \subset U$. Put $\mathscr{G}_{n}=\{g(n, x): x \in X\}$ for $n \in \mathbb{N}$. Then $\left\{s t^{2}\left(x, \mathscr{G}_{n}\right)\right\}=g^{4}(n, x)$, so $\left\{\mathscr{G}_{n}\right\}_{n \in \mathbb{N}}$ is a sequence of open covers such that $\left\{s t^{2}\left(x, \mathscr{G}_{n}\right): n \in \mathbb{N}\right\}$ is a local base at $x$ for all $x \in X$. Hence, by By the Moore Metrization theorem [1], $X$ is metrizable. This completes the proof.

COROLlARY 2.4. A space $X$ is metrizable if and only if there is a Heath $g$-function g such that

(1) if $x \in g(n, y)$ then $y \in g(n, x)$;

(2) $\left\{g^{2}\left(n, x_{n}\right): n \in \mathbb{N}\right\}$ is a local basis at $x$ for all $x \in X$.

THEOREM 2.5. A space $X$ is metrizable if and only if there is a Heath $g$-function $g$ such that

(1) if $x \in g(n, y)$ then $y \in g(n, x)$;

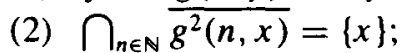

(3) if $\left\{x, x_{n}\right\} \subset g\left(n, y_{n}\right)$ then the sequence $\left\langle x_{n}\right\rangle$ has a cluster point. 
Proof. It is easy to prove necessity. To prove sufficiency, we need to prove that $x$ is a cluster point of the sequence $\left\langle x_{n}\right\rangle$. Let $q$ be a cluster point of $\left\langle x_{n}\right\rangle$. Suppose that $q \neq x$. Then there are infinitely many integer $m \geq n$ such that $x_{m} \in g(n, q)$. Now we have $\left\{x, x_{m}\right\} \subset g\left(n, y_{m}\right)$. By conditions (1) and (2) we get $x \in g\left(n, y_{m}\right)$ and $y_{m} \in g\left(n, x_{m}\right)$. Therefore, $\left\{x_{m}: m \geq n\right\} \subset g^{2}(n, x)$, so $q \in \overline{\left\{x_{m}: m \geq n\right\}} \subset \overline{g^{2}(n, x)}$. Thus $q \in \bigcap_{n \in \mathbb{N}} \overline{g^{2}(n, x)}=\{x\}$. Hence $q=x$, as required.

\section{Acknowledgement}

The author is grateful to Dr Chris Good for his kind help and valuable comments and suggestions on this paper, especially for the proof of Theorem 2.1. The author also thanks the referee for a number of suggestions which have improved the presentation of this paper.

\section{References}

[1] R. H. Bing, 'Metrization of topological spaces', Canad. J. Math. 3 (1951), 175-186.

[2] G. Gruenhage, 'Generalized metric spaces', in: Handbook of set-theoretic topology (North-Holland, Amsterdam, 1984) pp. 423-501.

[3] R. W. Heath, 'Arc-wise connectedness in semi-metric spaces', Pacific J. Math. 12 (1962), 1301-1319.

[4] R. Hodel, 'Moore spaces and $w \Delta$-spaces', Pacific J. Math. 38 (1971), 64 1-652.

[5] T. Ishii, 'On $w M$-spaces. I', Proc. Japan Acad. 46 (1970), 5-10.

Department of Mathematics and Statistics

College of Science

Sultan Qaboos University

P. O. Box 36, Al-Khodh 123

Muscat

Oman

e-mail:mohamad@squ.edu.om 
J. Aust. Math. Soc. 78 (2005) 